\title{
The "cost" of treating to target: cross-sectional analysis of patients with poorly controlled type 2 diabetes in Australian general practice
}

\author{
John Furler ${ }^{1 *}$, Justin W S Hii ${ }^{2}$, Danny Liew ${ }^{2}$, Irene Blackberry ${ }^{1}$, James Best ${ }^{3}$, Leonie Segal ${ }^{4}$ and Doris Young ${ }^{1}$
}

\begin{abstract}
Background: To describe the current treatment gap in management of cardiovascular risk factors in patients with poorly controlled type 2 diabetes in general practice as well as the associated financial and therapeutic burden of pharmacological treatment.

Methods: Cross-sectional analysis of data from the Patient Engagement and Coaching for Health trial. This totalled 473 patients from 59 general practices with participants eligible if they had HbA1c > 7.5\%. Main outcome measures included proportions of patients not within target risk factor levels and weighted average mean annual cost for cardiometabolic medications and factors associated with costs. Medication costs were derived from the Australian Pharmaceutical Benefits Schedule.

Results: Average age was 63 (range 27-89). Average HbA1c was $8.1 \%$ and average duration of diabetes was 10 years. $35 \%$ of patients had at least one micro or macrovascular complication and patients were taking a mean of 4 cardio-metabolic medications. The majority of participants on treatment for cardiovascular risk factors were not achieving clinical targets, with $74 \%$ and $75 \%$ of patients out of target range for blood pressure and lipids respectively. A significant proportion of those not meeting clinical targets were not on treatment at all. The weighted mean annual cost for cardiometabolic medications was AUD\$1384.20 per patient (2006-07). Independent factors associated with cost included age, duration of diabetes, history of acute myocardial infarction, proteinuria, increased waist circumference and depression.

Conclusions: Treatment rates for cardiovascular risk factors in patients with type 2 diabetes in our participants are higher than those identified in earlier studies. However, rates of achieving target levels remain low despite the large 'pill burden' and substantial associated fiscal costs to individuals and the community. The complexities of balancing the overall benefits of treatment intensification against potential disadvantages for patients and health care systems in primary care warrants further investigation.
\end{abstract}

Keywords: Type 2 diabetes, Cost, Treatment gap, Treatment burden

\section{Background}

Australia, like the rest of the world, is in the midst of an epidemic of diabetes. Over a million Australians have diabetes, of which over $85 \%$ is type 2 (T2DM) [1]. The prevalence more than doubled from 1989-90 to 2004-05 and is predicted to continue rising. Diabetes and its complications contributed $8.3 \%$ of the total burden of disease

\footnotetext{
* Correspondence: j.furler@unimelb.edu.au

'Department of General Practice, The University of Melbourne, Melbourne, Australia

Full list of author information is available at the end of the article
}

in Australia in 2003. It shortens life expectancy by up to 5 years and costs the community nearly $\$ 1$ billion annually in direct health care costs [2], a figure that is expected to treble over the next 40 years [3]. Future scenarios suggest a progressive rise in the human and health care costs associated with diabetes, particularly related to cardiovascular disease and the increasingly identified co-morbid depression.

Evidence-based clinical care focused on treating to targets for risk factors can improve outcomes for people with T2DM [4]. While patient-centred education and

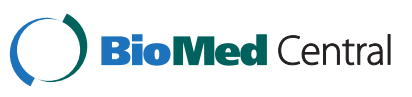


self-management interventions are important [5,6], pharmacotherapy is a key element of treatment for T2DM, both for glycaemic control and associated risk factors such as dyslipidaemia and hypertension. Targeting treatment to high risk patients is felt to be important [7].

In Australia, in line with other developed countries, there has been significant investment in programs to improve quality of clinical care and outcomes for diabetes. Over the last two decades, these have included the National Divisions Diabetes Program [8], the Australian Primary Care Collaboratives [9], the National Integrated Diabetes Program and targeted incentive payments to GPs and practices [10]. The Diabetes Care Project is currently underway, to trial a system of voluntary capitation payments to fund care for patients with diabetes in general practice, with the aim of improving comprehensive high quality care and reducing downstream costs (www. dcp.org.au).

A decade ago, early in the life of these initiatives, the gap between treatment goals and their translation into clinical practice remained wide. An Australian study reporting 2002 data suggested that patients with known diabetes identified in Australian general practices had poorly controlled disease and associated cardiovascular risk factors [11]. That study identified $48 \%, 88 \%$ and $74 \%$ of patients out of target for HbA1C, cholesterol levels and blood pressure (BP), respectively. The authors concluded that GPs needed to be more active, particularly in targeting treatment to patients with higher risk. While targets vary from country to country, these results are broadly comparable to those reported in the UK and US at that time, both in published studies and in analysis of national Quality and Outcomes Framework (QOF) data $[12,13]$.

We report here more recent cross-sectional data from the Patient Engagement and Coaching for Health (PEACH) study, a cluster randomised controlled trial of practice nurse-led telephone coaching for patients with poorly controlled T2DM in Australian general practices [14]. Our primary objective is to describe the treatment gap in management of cardiovascular risk factors, both in the initiation, as well as the intensification, of treatment to achieve targets. Our secondary objective is to describe the significant 'pill burden' borne by patients along with the associated financial cost and factors associated with this cost.

\section{Methods}

The PEACH study has been described elsewhere [14]. Practices were recruited from across Victoria via mailouts and newsletter promotions through Divisions of General Practice, and patients were identified from practice electronic databases or local pathology services. Patients with type 2 diabetes were eligible if their most recent $\mathrm{HbA} 1 \mathrm{c}$ was within the last 12 months and was greater than $7.5 \%$. Patients were recruited sequentially over 18 months through to August 2008. Baseline data for each patient was collected within 4 weeks of recruitment.

Practice nurses (PNs) collected data via face-to-face interviews. Height and weight were collected by the PN according to standard instructions. BP was recorded using usual clinical practice. The nurses also extracted data from the patients' medical records, including information on complications and current medication use. Data on medication use was based on what was prescribed by the GP and was recorded in medical record at the practice. PNs were asked to confirm drug usage on the list and also check for any additional complimentary medications taken. The PN then arranged baseline pathology testing $(\mathrm{HbA} 1 \mathrm{c}$, lipids, renal function and urinalysis). All the pathology laboratories used HbA1c assay methods aligned with DCCT [15] and undertook quality assurance for HbA1c and other biochemical assays.

The PEACH study adopted target levels for risk factors from the diabetes clinical guidelines in use in general practice at the time [16] and from the National Heart Foundation (Table 1).

The primary outcome of the PEACH trial was HbA1c level at 18 months post baseline. For $80 \%$ power and 5\% significance level (two-sided test), 464 patients (8 patients per practice) from 58 primary care practices were required (232 per group) to detect an absolute $0.5 \%$ reduction in mean HbA1c between the intervention and control groups. Sample size was based on a two-sample $\mathrm{t}$-test assuming a standard deviation of 1.44 and was inflated by 1.3 to allow for the correlation of the patient outcomes belonging to the same practice (assuming an intra-cluster correlation of 0.05 and variable sample cluster sizes) and allows for a $20 \%$ attrition rate over 18 months. A flow chart of patient recruitment is shown in Figure 1.

The PEACH study was funded by the National Health and Medical Research Council (NHMRC) General Practice Clinical Research Grants program. Ethics approval was granted by the University of Melbourne Human Research Ethics Committee. The trial is registered with Current Controlled Trials (ISRCTN 50662837).

\section{Medication cost estimation}

Medications were classified into the following groups: cardiovascular (antihypertensive, antiarrhythymic, antianginal, lipid lowering, inotropic, anticoagulants and antiplatelets), diabetic (oral hypoglycaemics and insulin), antidepressants and 'other'. Cardiometabolic medications encompassed both cardiovascular and diabetic medications. The use of antidepressant medications was used as a surrogate marker for the presence of depression. We 
Table 1 Targets for telephone coaching

\begin{tabular}{|c|c|}
\hline Clinical measure & Target \\
\hline $\mathrm{HbA1c}$ & $<6.5 \%$ \\
\hline Total cholesterol & $<4.0 \mathrm{mmol} / \mathrm{L}$ \\
\hline LDL cholesterol & $<2.0 \mathrm{mmol} / \mathrm{L}$ \\
\hline $\mathrm{BP}$ if microalbuminuria absent & $<130 / 80$ \\
\hline $\mathrm{BP}$ if microalbuminuria present & $<125 / 75$ \\
\hline Renal protection & $\begin{array}{l}\text { All patients with microalbuminuria } \\
\text { to be on angiotensin converting } \\
\text { enzyme inhibitor (ACEi) or angiotensin II } \\
\text { receptor blocker (ARB) unless } \\
\text { contraindicated }\end{array}$ \\
\hline Anti-thrombotic & $\begin{array}{l}\text { All patients to be on anti-platelet agent } \\
\text { unless contraindicated }\end{array}$ \\
\hline
\end{tabular}

estimated the costs of cardiovascular, diabetic and antidepressant medications only.

Medication costs were based on reimbursed prices in the Australian Pharmaceutical Benefits Schedule (PBS) in 2006-7. These were the costs met by the Australian Government which funds $84 \%$ of the costs of benefitpaid pharmaceuticals in Australia, (and 52\% of the costs of all medications). They do not include co-payments by consumers. The estimation of costs was based on the method adopted in another Australian study [17]. In summary, for each therapeutic class, a weighted average daily cost was estimated by calculating the sum-product of the cost of each drug dose and its proportional contribution to the class based on the market share as listed on the PBS. For example, assume that two drugs existed in a class and cost $\$ 100$ and $\$ 200$ per year, and comprised $60 \%$ and $40 \%$ of the market share of the class, respectively. The weighted average cost of that class would be $\$ 100 * 60 \%+\$ 200 * 40 \%=\$ 140$ per year. The various doses of the drug, costs and services supplied were sourced from the PBS based on the period September 2006-Sept 2007 [18]. Where a particular drug was available in different doses, we assumed that the doses used were those recommended in treatment guidelines [19-22]. The underlying assumption with the above method for cost estimation is that patients were fully compliant with their medications.

\section{Statistical analyses}

Descriptive analyses were undertaken on the baseline patient data from the PEACH study.

Statistical analyses for assessing treatment gap were performed using SPSS for Windows version 19.0 (IBM Corp., New York, NY). To determine proportions of patients not within target risk factor levels, risk factor prevalence data were cross-tabulated against data on relevant medication use. Statistical analyses for the costing of medications were performed using PASW for Mac version 18.0. First, a series of univariate linear regression analyses were undertaken to identify significant factors associated with cardiovascular and diabetes (cardiometabolic) medication costs. Significant variables from these analyses were then entered into a multivariate linear regression model to identify independent factors associated with cardio-metabolic medication costs. $\mathrm{P}$ values of less than 0.05 were considered statistically significant.

\section{Results}

The baseline characteristics of participants are summarised in Tables 2 (demographic) and 3 (clinical). Age ranged from 27-89 years. 13 patients had a diabetes duration of less than 1 year recorded. Over one third (34\%) of participants had a record in the medical file

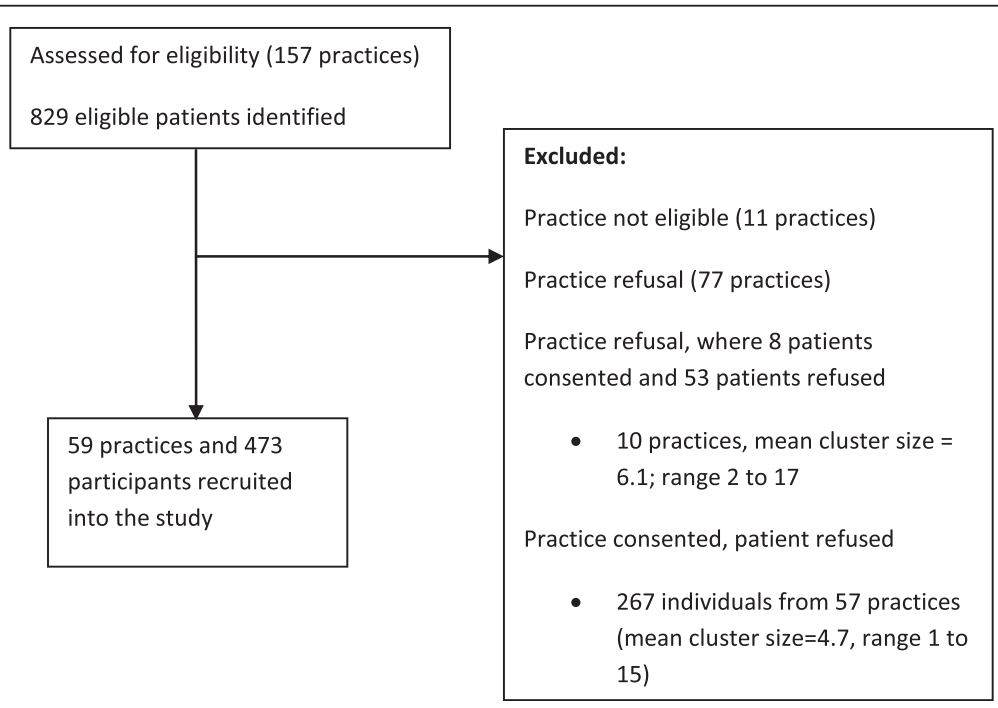

Figure 1 Flowchart of participant recruitment. 
Table 2 Demographics of participants $(n=473)$

Age (years) Mean (SD)
Sex (\%) Male
Smoker (\%)
Current
Highest Level of Education (\%)
Primary
Completed Secondary School
Trade or TAFE
Completed University Degree
Employment Status (\%)
Employed
Unemployed
Home Duties
Retired
Health Care Card (\%)

of at least one diabetes complication (micro and/or macrovascular).

The mean number of cardiometabolic medications were 4.2 (SD 2.2) with a range of $0-13$. The mean number of total medications were 7.5 (SD 4.1) with a range of $1-24.33 .2 \%$ of participants were prescribed antidepressant medications at antidepressant doses. The proportions of medications are shown in Table 4.
Table 4 Proportions of cardiometabolic medications

\begin{tabular}{ll}
\hline Medication & Frequency \\
\hline Hypoglycaemic & \\
Insulin & $23 \%(109 / 473)$ \\
Metformin & $76.7 \%(415 / 473)$ \\
Other oral Hypoglycaemics & $87.7 \%(415 / 473)$ \\
Hypolipidaemic & $65.1 \%(308 / 473)$ \\
Statins & $62.8 \%(297 / 473)$ \\
Fibrate & $2.7 \%(13 / 473)$ \\
Ezetimibe & $4.2 \%(20 / 473)$ \\
Antihypertensives & $71.5 \%(338 / 473)$ \\
Beta blocker & $18.6 \%(88 / 473)$ \\
ACEi & $40.2 \%(190 / 473)$ \\
ARB & $33.8 \%(160 / 473)$ \\
Antiplatelets & $46.9 \%(222 / 473)$ \\
Aspirin & $44.2 \%(209 / 473)$ \\
Dipyridamole & $1.3 \%(6 / 473)$ \\
Clopidogrel & $4.9 \%(23 / 473)$ \\
Warfari & $5.9 \%(28 / 473$ \\
\hline
\end{tabular}

Table 5 shows the prevalence of cardiovascular risk factors. Our sample had high prevalence of hypertension and dyslipidaemia, with $74 \%$ and $75 \%$ being out of target range for BP and lipids, respectively. Significant proportions of participants with a risk factor were not on

Table 3 Clinical characteristics of participants

\begin{tabular}{|c|c|c|c|c|}
\hline Diabetic characteristics & Mean (SD) & Median & Range & Inter-quartile Range \\
\hline $\mathrm{HbA1c}(\%)$ & $8.1(1.3)$ & 7.8 & $5.3-16.0$ & $7.3-8.6$ \\
\hline Diabetes Duration (years) & $10(7)$ & 10 & $0-44$ & $5-14$ \\
\hline Systolic BP (mmHg) & $138(18)$ & 138 & $90-221$ & $126-149$ \\
\hline Diastolic BP (mmHg) & $79(10)$ & 80 & $53-115$ & $72-85$ \\
\hline Total Cholesterol (mmol/L) & $4.5(1.1)$ & 4.4 & $2.5-12.4$ & $3.7-5.0$ \\
\hline Waist Circumference (cm) & $109(15)$ & 108 & $68-167$ & $99-118$ \\
\hline $\mathrm{BMI}\left(\mathrm{kg} / \mathrm{m}^{2}\right)$ & $32.8(6.5)$ & 31.7 & $19.8-54.5$ & $28.1-36.2$ \\
\hline Medications (total) & $7.5(4.1)$ & 7 & $1-24$ & $5-10$ \\
\hline Medications (Cardio-metabolic) & $4.2(2.2)$ & 4 & $0-13$ & $3-5$ \\
\hline Diabetes complications & Rate & & & \\
\hline Any & $34.1 \%(155 / 434)$ & & & \\
\hline \multicolumn{5}{|l|}{ Macro } \\
\hline AMI & $16.8 \%$ & & & \\
\hline CVA & $5.5 \%$ & & & \\
\hline PVD & $2.5 \%$ & & & \\
\hline \multicolumn{5}{|l|}{ Micro } \\
\hline Retinopathy & $20.7 \%$ & & & \\
\hline Neuropathy & $6.6 \%$ & & & \\
\hline Nephropathy & $12.4 \%$ & & & \\
\hline
\end{tabular}


Table 5 Risk factor prevalence and treatment

\begin{tabular}{lll}
\hline Risk factor & Prevalence $\mathbf{n} / \mathbf{N}(\%)^{*}$ & $\begin{array}{l}\text { Number (\%) of patients with } \\
\text { risk factor not on treatment }\end{array}$ \\
\hline BP out of target range & $341 / 460(74)$ & $86(25)$ \\
Lipids out of target range & $319 / 424(75)$ & $128(40)$ \\
Microalbuminuria or albuminuria present & $122 / 428(29)$ & 35 (27) (ACEi or ARB) \\
Thrombotic risk present (assumed to be all participants) & $473 / 473(100)$ & 250 (53) (antiplatelet) \\
\hline
\end{tabular}

* Risk factor prevalences have differing denominators due to some missing data.

treatment (see right hand column of Table 5). Most participants $(73 \%)$ with albuminuria were taking angiotensin converting enzyme inhibitors (ACEi) and/or angiotensin receptor blockers (ARB). Lastly, only $47 \%$ of the sample was taking anti-platelet agents.

Figures 2 and 3 illustrate the overall distribution of patients achieving target and on treatment for BP and lipids. Among patients who were on treatment for a risk factor, the majority remained out of target: $76 \%$ of patients on antihypertensive treatment and $70 \%$ of those on lipid-lowering treatment.

The mean average weighed cost per year for these medications was AUD\$1384 (SD \$850). Increasing age, greater duration of diabetes, a history of acute myocardial infarction (AMI), depression and the presence of proteinuria were independent factors associated with cardio-metabolic medication costs (Table 6).

Costs were significantly lower for patients without any recorded diabetes complications (Table 7).

\section{Discussion}

Cardiovascular disease is the main cause of death for people with T2DM, but with multi-factorial clinical interventions, significant improvements in absolute cardiovascular risk can be achieved [7]. Our primary objective was to describe the treatment gap in management of cardiovascular risk factors, both in the initiation, as well as the intensification of treatment to achieve targets. Our

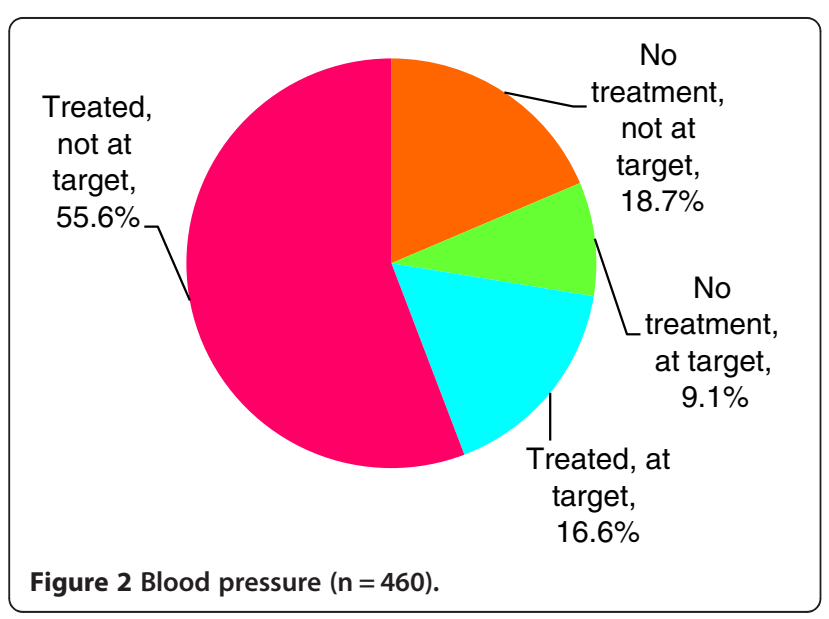

findings identify that among patients with poorly controlled T2DM in Australian general practice, the majority are on treatment for associated cardiovascular risk factors.

We identified higher rates of treatment compared to a decade ago in Australia [11]. This improvement may represent the influence of guidelines and incentive schemes and initiatives, although it may also represent selection of a group with more severe T2DM, as our patients were selected with HbA1c above 7.5\%. However, despite the higher rates of treatment, our data shows that substantial numbers remain untreated, contrary to evidence-based guidelines. Our study also identified that the majority of treated patients were not achieving targets for BP and lipids, a finding in accord with earlier studies $[11,23]$. Similar findings were identified in the UK [12] where rates of being "in-target" for BP and cholesterol amongst patients with type 2 diabetes were $36-70 \%$ and $41-72 \%$ respectively (although targets used in these studies were higher than those used in the current study). Similar findings were identified in the US $[13,24]$.

Our secondary objective was to describe the number of medications participants were taking together with estimated costs associated with pharmacological treatment from the perspective of the Australian government. We found a high "pill burden" amongst our participants. While there is international population level data about trends to increasingly intense, complex and costly

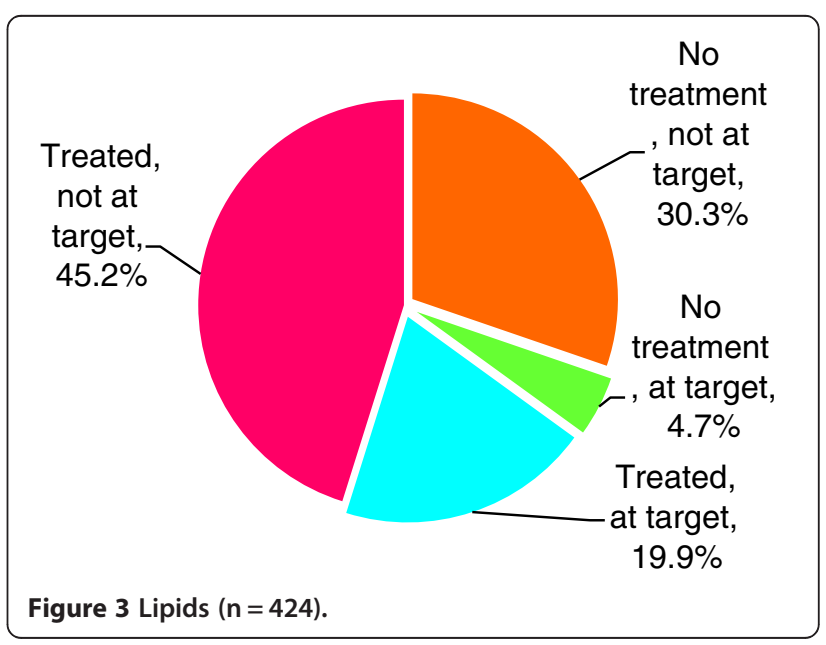


Table 6 Weighted average mean and independent predictors of cardiometabolic medication costs

\begin{tabular}{llll}
\hline Variable & $\begin{array}{l}\text { Mean cost } \\
\text { variance }(\mathbf{S A U})\end{array}$ & $\mathbf{p}$ value & $\mathbf{9 5 \%} \mathbf{C l}$ \\
\hline AMl & $+1,018.45$ & 0.000 & $542.88-1494.02$ \\
Depression & +486.45 & 0.000 & $320.73-652.16$ \\
Proteinuria & +215.10 & 0.018 & $36.76-393.44$ \\
$\begin{array}{l}\text { Duration of diabetes } \\
(+1 \text { year) }\end{array}$ & +18.43 & 0.001 & $7.29-29.57$ \\
Age (+ 1 year) & +8.18 & 0.040 & $0.36-16.00$ \\
Waist Circumference & +7.45 & 0.005 & $2.21-12.69$ \\
\hline
\end{tabular}

treatment regimes for diabetes [25,26], there is very little data published on the medication burden of individual patients. A study of 128 patients in the US [27] found they were taking a mean of 4.1 medications to control diabetes and an overall mean pill burden of 5.8 different medications. A study of 1,991 US primary care patients with a HbA1c of $9.1+/-1.3$ found they were taking a mean of 9.1 medications in total [28]. Australian data suggests that amongst all people with diabetes less than $1 \%$ use four or more cardiometabolic medications whereas this was the average figure in our study [29].

Pharmaceuticals comprise one of the fastest growing costs within the health care systems. In Australia medications for the treatment of cardiovascular disease and diabetes are significant contributors to total PBS cost [18]. Given the rapidly rising prevalence of T2D and continued poor glycaemic control, the estimated mean average weighted cost per year for cardiometabolic medications of more than AUD\$1384 (SD \$850) per individual represents a considerable encumbrance on the PBS system. Predictably, a history of AMI was the most significant independent factor associated with pharmaceutical cost, increasing costs by over AUD $\$ 1000$ annually. Any complication added significantly to medication costs. Importantly, depression also independently contributed to costs of medications, with the excess cost associated being almost half that of AMI (\$AUD486 annually). Part of the reason for this increased cost was that patients with depression used more cardio-metabolic medications (mean 5.4 vs $3.6, \mathrm{p}<0.0001$ ).

In the context of two decades of initiatives and incentive schemes aimed at improving diabetes outcomes, is this apparent failure on the part of GPs and patients to embrace more intensive pharmacological treatment

Table 7 Cost of cardiometabolic medications for those with and without complications

\begin{tabular}{llll}
\hline Complication & $\mathbf{N}$ & Mean (AUD) & SD \\
\hline None & 307 & 1235.80 & 811.80 \\
Any & 166 & 1658.67 & 851.65 \\
\hline
\end{tabular}

$\mathrm{p}$ value for difference $<0.001$. for T2D simply another example of clinical inertia $[28,30,31]$ ? From a clinical perspective, this might suggest that new strategies such as care coordinators or coaches [32] are needed to drive further intensification of treatment. After all, if patients are to be prescribed medications, they ought reasonably to expect clinical benefit from them. Our findings may also suggest the need for renewed effort in detecting and managing depression in people with diabetes. We need open discussion of the cost of such measures and the implications for sustainability of the health system.

However, our findings may also reflect collective views of GPs and patients about the primary aim of care for the patient with diabetes. There is debate about the diminishing clinical benefit and increasing personal and lifestyle costs associated with the relentless pursuit of surrogate markers in diabetes and cardiovascular disease to the exclusion of hard endpoints that matter to patients [33,34]. Similar issues have been raised in relation to the pharmacological management of depression [35]. GPs may be sensitive to this issue in their close relationships with patients, aware of the burden of treatment that patients bear and are reluctant to add to it [36]. Our findings may reflect decisions made by GPs and their patients to limit their pursuit of biological markers at the cost of quality of life and other patient-centred outcomes. This may reflect an attempt by GP and patient to say "enough is enough", to tailor clinical decisions to the personal context of the patient. This attention to the way treatment can add complexity and "work" to the daily lives and circumstances of patients living with chronic conditions is an attempt to recast the issue of "non-compliance". Practitioners and patients in primary care may both be implicitly engaging in what has been called "minimally disruptive medicine" [37], balancing what is realistic and achievable for patients against evidence based guidelines driven by disease markers.

Understanding how patients and health practitioners engage in this work, how they interpret evidence and guidelines while incorporating outcomes of importance to patients warrants much more investigation. Development of robust measures of patient-centred outcomes to allow their incorporation into guidelines in ways that account for complexity, multiple morbidity and social context, is a key area of research if evidence is to be relevant to the realities of practice [38].

One important limitation of our study is selection bias. Patients were recruited into the study on the basis of poor glycaemic control. This may have led to a sample of patients with poorer control of other cardiovascular risk factors. Our results cannot be transferred to type 2 diabetes patients in general, but are only interpretable for this selected group. Another potential limitation is the reliability of documentation of complications in the 
participants' medical records. Complication rates were determined by the PN from the medical record based on recorded diagnoses. There may be significant variation between practices in the documentation of individual complication rates. We were also unable to perform quality checks on the data extraction by PNs. This may have led to underreporting of complications. Because we assumed no contraindications to treatment, the extent of under-treatment is likely to have been over-estimated. Our use of antidepressant medications at antidepressant doses as a surrogate marker for depression may be an overestimate as some patients may be on treatment for other conditions such as anxiety. On the other hand, we did not count those with depression but not on pharmacotherapy. Our costing was estimated and prices may vary between non-concessional patients. In addition, it is very difficult to estimate costs due to private pharmacy costs, which can vary enormously. However, as $62 \%$ of our patients were receiving health care card benefits, our costing was likely to have been an underestimate. Finally, the underlying assumption underpinning annual costs was that patients remained compliant with their medication throughout the year. Despite its limitations, participation of a large sample of primary care patients across Victoria is a major strength of our study, as is the collection of detailed patients' medications data.

\section{Conclusion}

Despite significant investment of money and resources at system and practice level, and modest improvement in treatment rates for cardiovascular risk factors in patients with type 2 diabetes in Australian general practice, there is still opportunity to intensify treatment to achieve target levels. We did find evidence that greater intensity of treatment is directed to those with higher risk. More patients are on now treatment and for the majority of patients, the pill burden continues to rise, at significant cost to funders, while improved disease markers and outcomes remain difficult to achieve. The challenge is to understand better how practitioners and patients balance evidence with the complex realities of practice and to develop and include measures within guidelines that reflect important patient priorities.

\section{Competing interests}

The authors declare that they have no competing interests.

\section{Authors' contributions}

DY and JB conceptualised the research. DY, JB, JF and LS conceived the development of study design. DY, IB and JF oversaw the running of the trial. $J F, J H, D L$ drafted the manuscript. JH, DL, JF and IB conducted the data analysis. All authors read and approved the final manuscript.

\section{Acknowledgments}

The PEACH study was supported by the Australian National Health and Medical Research Council (NHMRC) (ID 359374 and 566586). We would like to thank the divisions, General Practitioners, Practice Nurses and patients participating in the study.

\section{Funding}

The study is funded by the National Health and Medical Research Council (NHMRC) General Practice Clinical Research Grants program.

\section{Author details}

'Department of General Practice, The University of Melbourne, Melbourne, Australia. ${ }^{2}$ Melbourne EpiCentre, Royal Melbourne Hospital, The University of Melbourne, Melbourne, Australia. ${ }^{3}$ Department of Medicine, St Vincent's Hospital, The University of Melbourne, Melbourne, Australia. ${ }^{4}$ Division of Health Sciences, University of South Australia, Adelaide, Australia.

Received: 1 August 2012 Accepted: 26 February 2013

Published: 8 March 2013

\section{References}

1. Australian Institute of Health and Welfare: Diabetes: Australian facts. Canberra: AlHW2008; 2008.

2. Dunstan D, Zimmett $P$, Welborn T: The final report of the Australian diabetes, obesity and lifestyle study (AusDiab). Melbourne: International Diabetes Institute; 2001.

3. Davis WA, Knuiman MW, Hendrie D, Davis TME: The obesity-driven rising costs of type 2 diabetes in Australia: projections from the Fremantle diabetes study. Intern Med J 2006, 36(3):155-161.

4. National Health Priority Action Council (NHPAC): National chronic disease strategy. Canberra: Australian Government Department of Health and Ageing; 2006.

5. Norris S, Lau J, Smith S, Schmid C, Engelgau M: Self-management education for adults with type 2 diabetes: a meta-analysis of the effect on glycemic control. Diabetes Care 2002, 25(7):1159-1171.

6. Ismail K, Winkley K, Rabe-Hesketh S: Systematic review and meta-analysis of randomised controlled trials of psychological interventions to improve glycaemic control in patients with type 2 diabetes. Lancet 2004, 363(9421):1589-1597. 2004/5/15.

7. Gaede P, Lund-Andersen H, Parving H-H, Pedersen O: Effect of a multifactorial intervention on mortality in type 2 diabetes. $N$ Engl J Med 2008, 358(5):580-591.

8. Bonney MA, Carter S, Burns J, Powell-Davies G, Harris MF: National divisions diabetes program, data collation project, volume 3, divisions of general practice - diabetes profiles. Quality of care and health outcomes - collated division data. Sydney: Centre for GP Integration Studies, School of Community Medicine, University of New South Wales; 2000.

9. Improvement Foundation: Austalian primary care collaboratives program. 2012. Accessed July 23 2012]; Available from: http://www.apcc.org.au/.

10. Australian Government Department of Human Services: Practice incentives program diabetes incentive guidelines-July 2012. 2012 [25 July 2012].. Available from: http://www.medicareaustralia.gov.au/provider/incentives/ pip/files/9520-1206en.pdf.

11. Wan Q, Harris MF, Jayasinghe UW, Flack J, Georgiou A, Penn DL, et al: Quality of diabetes care and coronary heart disease absolute risk in patients with type 2 diabetes mellitus in Australian general practice. Qual Saf Health Care 2006, 15(2):131-135.

12. Khunti K, Gadsby R, Millett C, Majeed A, Davies M: Quality of diabetes care in the UK: comparison of published quality-of-care reports with results of the quality and outcomes framework for diabetes. Diabet Med 2007, 24(12):1436-1441.

13. Crosson JC, Ohman-Strickland PA, Campbell S, Phillips RL, Roland MO,

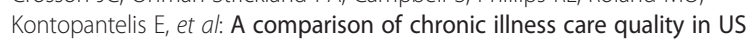
and UK family medicine practices prior to pay-for-performance initiatives. Fam Pract 2009, 26(6):510-516.

14. Young D, Furler J, Vale M, Walker C, Segal L, Dunning P, et al: Patient engagement and coaching for health: the PEACH study - a cluster randomised controlled trial using the telephone to coach people with type 2 diabetes to engage with their GPs to improve diabetes care: a study protocol. BMC Fam Pract 2007, 8(20).

15. The DCCT Research Group: Feasibility of centralized measurements of glycated hemoglobin in the Diabetes Control and Complications Trial: a multicenter study. Clin Chem 1987, 33(12):2267-2271. 
16. Harris $P$, Mann $L$, Phillips $P$, Snowdon $T$, Webster C: Diabetes management in general practice: 12th edition. Melbourne: Diabetes Australia and the Royal Australian College of General Practitioners; 2005.

17. Ademi Z, Liew D, Chew D, Conner G, Shiel L, Nelson M, et al: Drug treatment and cost of cardiovascular disease in Australia. Cardiovasc Ther 2009, 27:164-172.

18. Commonwealth of Australia: Pharmaceutical benefits schedule item reports. Period September 2006 to September 2007.. Accessed 11 May 2012; Available from: https://www.medicareaustralia.gov.au/statistics/ pbs_group.shtml.

19. Brunner EJ, Marmot M: Social organisation, stress and health. In Social determinants of health. 2nd edition. Edited by Marmot MG, Wilkinson RG. Oxford; New York: Oxford University Press; 2006:6-30.

20. Rossi S (Ed): Australian Medicines Handbook 2013. Adelaide: Australian Medicines Handbook Pty Ltd; 2013.

21. Expert Group Psychotropic: Therapeutic guidelines (Psychotropics). Melbourne: Therapeutic Guidelines Limited; 2008.

22. National Heart Foundation of Australia and the Cardiac Society of Australia and New Zealand: Position statement on lipid management. Heart Lung Circ 2005, 14(4):275-291.

23. Thomas MC, Nestel PJ: Management of dyslipidaemia in patients with type 2 diabetes in Australian primary care. Med J Aust 2007, 186(3):128-130.

24. McFarlane SI, Jacober SJ, Winer N, Kaur J, Castro JP, Wui MA, et al: Control of cardiovascular risk factors in patients with diabetes and hypertension at urban academic medical centers. Diabetes care 2002, 25(4):718-723.

25. Alexander GC, Sehgal NL, Moloney RM, Stafford RS: National trends in treatment of type 2 diabetes mellitus, 1994-2007. Arch Intern Med 2008, 168(19):2088-2094. 2008.

26. The Health and Social Care Information Centre PaPCS: Prescribing for Diabetes in England: 2005/6 to 2011/12. London: NHS Information Centre; 2012.

27. Grant RW, Devita NG, Singer DE, Meigs JB: Polypharmacy and medication adherence in patients with type 2 diabetes. Diabetes care 2003, 26(5):1408-1412. 2003.

28. Shah BR, Hux JE, Laupacis A, Zinman B, van Walraven C: Clinical inertia in response to inadequate glycemic control: do specialists differ from primary care physicians? Diabetes Care 2005, 28(3):600-606. 2005

29. AlHW, Webbie K, O'Brien K: Use of medicines by Australians with diabetes. Canberra: AlHW; 2006.

30. Parchman ML, Pugh JA, Romero RL, Bowers KW: Competing demands or clinical inertia: the case of elevated Glycosylated hemoglobin. Ann Fam Med 2007, 5(3):196-201. 2007.

31. Wolpert HA, Anderson BJ: Management of diabetes: are doctors framing the benefits from the wrong perspective? BMJ 2001, 323(7319):994-996. 2001.

32. Vale M, Sundararajan V, Best J, Jelinek M: Four year follow-up of the multicentre RCT of the $\mathrm{COACH}$ study shows that the $\mathrm{COACH}$ Programme keeps patients out of hospital. Eur Heart J 2005, 26:501-502.

33. Yudkin JS, Lipska KJ, Montori VM: The idolatry of the surrogate. Br Med J 2011, 343:d7995

34. Yudkin JS, Richter B, Gale EAM: Intensified glucose lowering in type 2 diabetes: time for a reappraisal. Diabetologia 2010, 53(10):2079-2085.

35. Dowrick C: Beyond depression: a new approach to understanding and management. 2nd edition. Oxford: Oxford University Press; 2009.

36. Guthrie B, Inkster M, Fahey T: Tackling therapeutic inertia: role of treatment data in quality indicators. BMJ (Clinical research ed) 2007 335(7619):542-544. 2007.

37. May C, Montori VM, Mair FS: We need minimally disruptive medicine. Br Med J 2009, 339:b2803.

38. Valderas JM, Alonso J: Patient reported outcome measures: a modelbased classification system for research and clinical practice. Qual Life Res 2008, 17(9):1125-1135.

\section{doi:10.1186/1471-2296-14-32}

Cite this article as: Furler et al: The "cost" of treating to target: crosssectional analysis of patients with poorly controlled type 2 diabetes in Australian general practice. BMC Family Practice 2013 14:32.

\section{Submit your next manuscript to BioMed Central and take full advantage of:}

- Convenient online submission

- Thorough peer review

- No space constraints or color figure charges

- Immediate publication on acceptance

- Inclusion in PubMed, CAS, Scopus and Google Scholar

- Research which is freely available for redistribution

Submit your manuscript at www.biomedcentral.com/submit

C Biomed Central 